\title{
Analysis of the performances of the Covid-19 therapeutic approaches in the United Arab Emirates
}

\author{
Alberto Boretti ${ }^{1, *}$
}

${ }^{1}$ Deanship of Research, Prince Mohammad Bin Fahad University, Al

Khobar, Saudi Arabia

\section{*Correspondence}

a.a.boretti@gmail.com

(Alberto Boretti)

\begin{abstract}
Objectives: To evaluate the performances of the therapeutic approaches adopted in the United Arab Emirates to fight Covid-19 infection.

Methods: Statistical analysis of the number of cases and fatalities for the United Arab Emirates. The fatality rates are compared to those of reference countries adopting different approaches.

Results: The United Arab Emirates outperformed the reference countries with a much smaller number of fatalities. While this number is not only driven by therapies, the different therapeutic approaches are the most important driver.

Conclusions: Combination therapies are most effective than single drug therapies. The use of antivirals especially in the early stages reduces the number of fatalities.
\end{abstract}

\section{Keywords}

Covid-19; Antivirals; Combination therapies

\section{Introduction}

The response to the Covid-19 pandemic has been very different across the world, and as a result, also the impact of Covid19 in terms of fatalities has been very different. There have been significant differences in terms of restrictions limiting the spread of the virus, as well as of treatment of the infected patients. Regarding treatment, in some countries, no antivirals are administered to asymptomatic or mild cases, and practically nothing more than corticosteroids are given to severe and critical cases, in addition to oxygen or ventilation. There are other countries where antivirals are given to asymptomatic patients in the risk categories in outpatient settings, as well as to mild, severe, and critical cases within hospital settings. The goal of this work is to highlight the different therapeutic approaches followed in the Arab States of the Gulf countries vs. those adopted in western countries. The antiviral drugs that are used in the Arab States of the Gulf countries are mostly discouraged in the western countries. This work aims to show differences in the number of cases, number of fatalities, and therapies and trying to correlate them.

\section{Method}

Information of the number of cases and fatalities, plus the number of tests and number of vaccines administered, has been obtained from ourworldindata.org. The CSV file downloaded from ourworldindata.org at the time of writing and containing all data used in the images and the statements about the number of cases, tests, fatalities, vaccines, are provided in the supplementary. Time series are shown when deemed necessary. Information on the therapies is obtained from publicly available links to health organizations.

\section{Results}

The latest percentages of infected are variables, but usually a few percent of the total population.

In the countries considered in Fig. 1.a (cumulative confirmed cases per 1,000,000 people), these percentages are variable from less than 0.1 to more than $6 \%$. The vast majority of the cases are mild or asymptomatic. Even the World Health Organization admits that $80 \%$ of the total cases are mild or asymptomatic. Hence, those infected and undetected may be many more of those recorded, depending on the specific country.

The cumulative fatalities are also variable significantly, in the cases of Fig. 1.b (cumulative fatalities per 1,000,000 people), from practically $0.00 \%$ to $0.17 \%$.

The cumulative case fatality rate (ratio between confirmed deaths and confirmed cases) of Fig. 1.c is variable from less than $0.1 \%$ to above $3.5 \%$.

Including the undetected mild or asymptomatic, the true cumulative fatality rate is less.

The therapeutic approach to Covid-19, which is one relevant, if not the most relevant, part of the response of a country, has been also very different in different countries. The therapeutic approach is partially an explanation of the observed different cumulative fatality rates. The cumulative fatality rate is also affected by other drivers such as geographical, demographic, social, economic, cultural, and policing.

Differences in therapies are relevant in early and mid-stages 

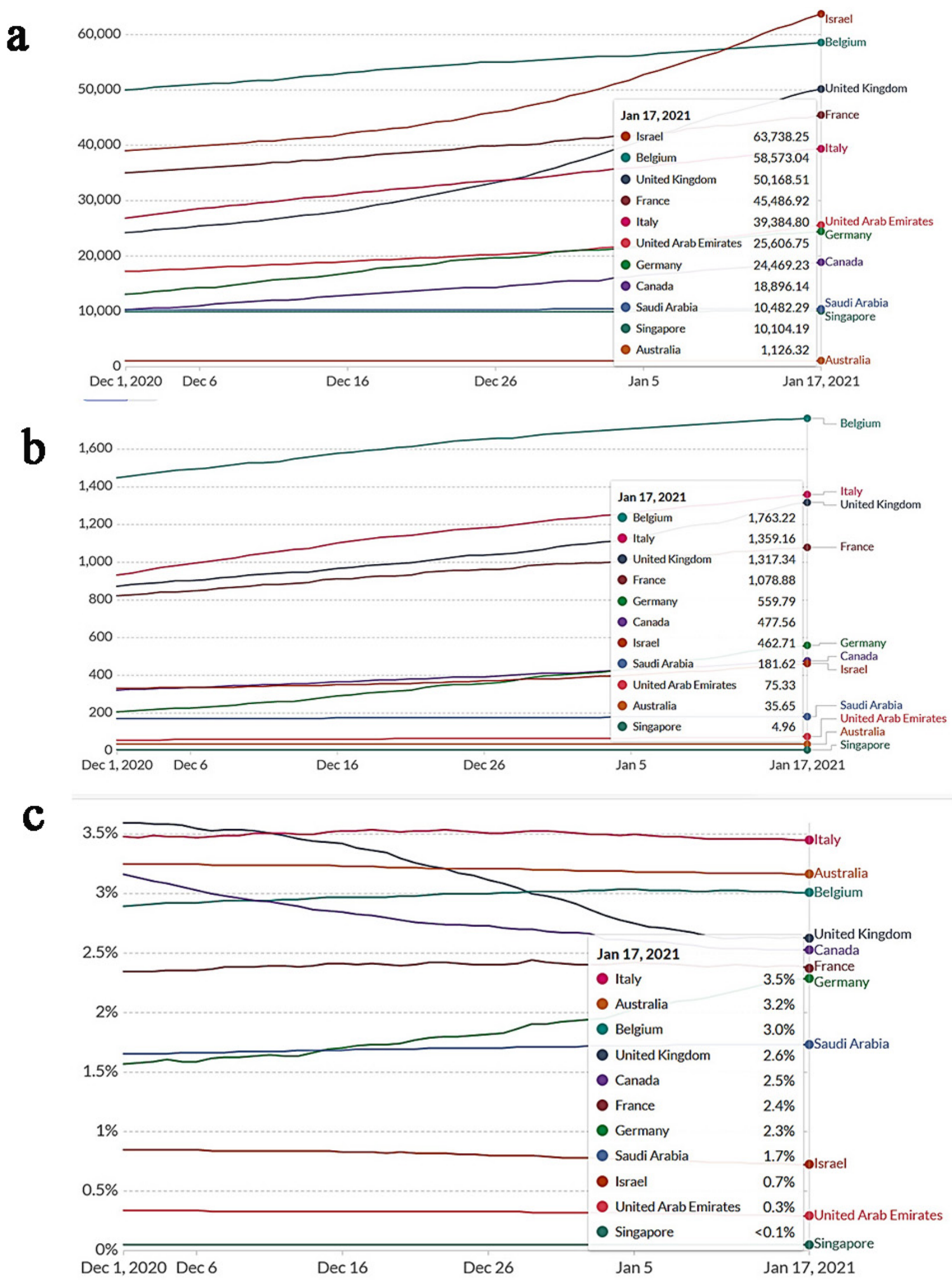

F I G U R E 1. Cumulative cases per million (a), cumulative fatalities per million (b) and the ratio of cumulative fatalities to cumulative cases (c) for selected countries. Images from ourworldindata.org. CC BY. 


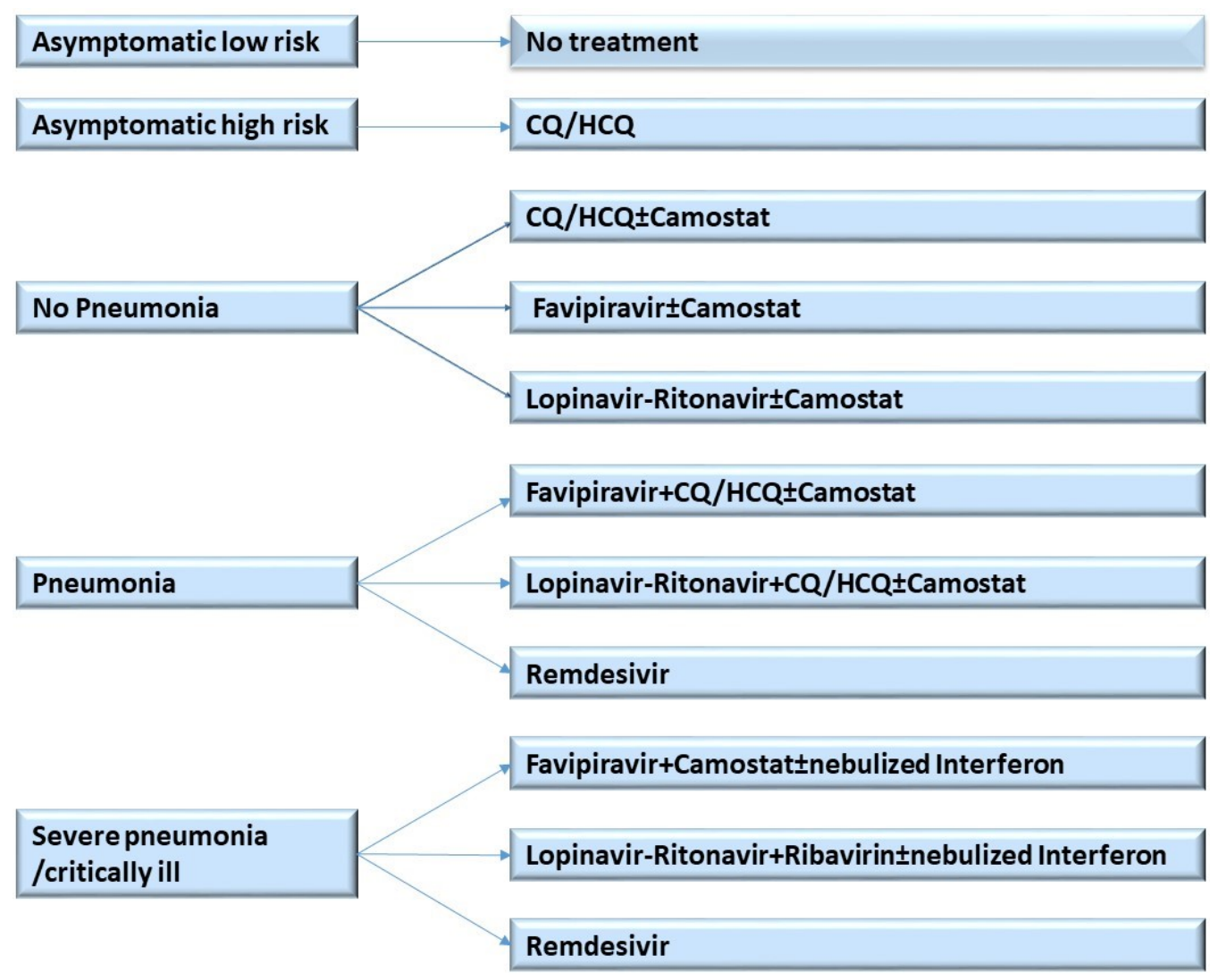

Interferon

F I G URE 2. Scheme of the therapeiutic options available in the UAE [4].

and medium to severe and critical cases.

While fatalities follow late-stages, it must be mentioned as the occurrence of late-stage infection is often the result of improper treatment during early stages, as the viral load buildup is determinant for Covid-19 infection fatalities [1-3].

\subsection{COVID-19 guideline in the United Arab Emirates}

The presence of pneumonia is frequent in medium to severe cases. Acute Respiratory Distress Syndrome (ARDS), the respiratory failure characterized by rapid widespread inflammation in the lungs often causing Covid-19 infection fatalities, is often present in critical cases. The way to address ARDS is first and foremost through earlier use of antivirals, i.e. targeting the primary cause of the pathology when it is easier. Without reaching very high viral loads, usually, patients do not have ARDS. Thus, early stages therapeutic interventions are determinant, more than late stages therapeutic interventions.

In addition to antivirals, other drugs that are targeting the immune system response, either in the direction of powering this response as well as calming the immune system, are also considered. Antibodies are also considered. Adjunctive therapies are also used to prevent or treat infection or complications.

Being the problem quite complex, different drugs may work better depending on the stage and the specific characteristics of the patient. Combination therapies may deliver better results.

According to [4], the proposed therapeutic regimens for adults in the United Arab Emirates (Abu Dhabi) are those summarized in Fig. 2. The details are given below, specific to the stage:

Asymptomatic Covid-19 cases usually receive no treatment, but if high risk, they are treated with Chloroquine (CQ) Phosphate $500 \mathrm{mg}$ PO (by mouth) BID (twice a day) X 2 doses, then $250 \mathrm{mg}$ PO BID or Hydroxychloroquine (HCQ) $400 \mathrm{mg}$ PO BID X 2 doses then $200 \mathrm{mg}$ PO BID. All these alternatives are a total of 5 days [4].

Covid-19 cases without pneumonia for 5 days receive HCQ 400mg PO BID then $200 \mathrm{mg}$ PO BID, or CQ Phosphate $500 \mathrm{mg}$ PO BID X 2 doses, then $250 \mathrm{mg}$ PO BID, or Favipiravir $1600 \mathrm{mg}$ PO BID X 2 doses, then $600 \mathrm{mg}$ PO BID, or Lopinavir-Ritonavir (200/50 mg) 2 tablets PO BID. All these alternatives are a total of 5 days. The addition of Camostat 200 $\mathrm{mg}$ PO TID (three times a day) X 5 days is optional [4].

Covid-19 cases with pneumonia for 7 days receive Favipiravir $1600 \mathrm{mg}$ PO BID X 2 doses then $600 \mathrm{mg}$ PO BID (total 7 days) + HCQ 40 0mg PO BID X 2 doses then $200 \mathrm{mg}$ PO BID (total 5 to 7 days) \pm Camostat $200 \mathrm{mg}$ PO TID for 5 to 7 days, same as before with CQ Phosphate $500 \mathrm{mg}$ PO BID $\mathrm{X} 2$ doses then $250 \mathrm{mg}$ PO BID (total 5 to 7 days) replacing HCQ, or Lopinavir-Ritonavir (200/50 mg) 2 tablets PO BID (total 7 days) + HCQ $400 \mathrm{mg}$ PO BID X 2 doses, then $200 \mathrm{mg}$ PO BID (total 5 to 7 days) \pm Camostat $200 \mathrm{mg}$ PO TID (5 to 7 days), same as before with CQ Phosphate $500 \mathrm{mg}$ PO BID X 2 doses, then $250 \mathrm{mg}$ PO BID) (5 to 7 days) replacing HCQ, or Remdesivir $200 \mathrm{mg}$ IV (Intravenous) on day 1, followed by $100 \mathrm{mg}$ IV daily. Interferon therapy is an option in cases of moderate disease [4].

Covid-19 cases with severe pneumonia/critically ill patients for 10 days receive Favipiravir 1600 mg PO BID X 

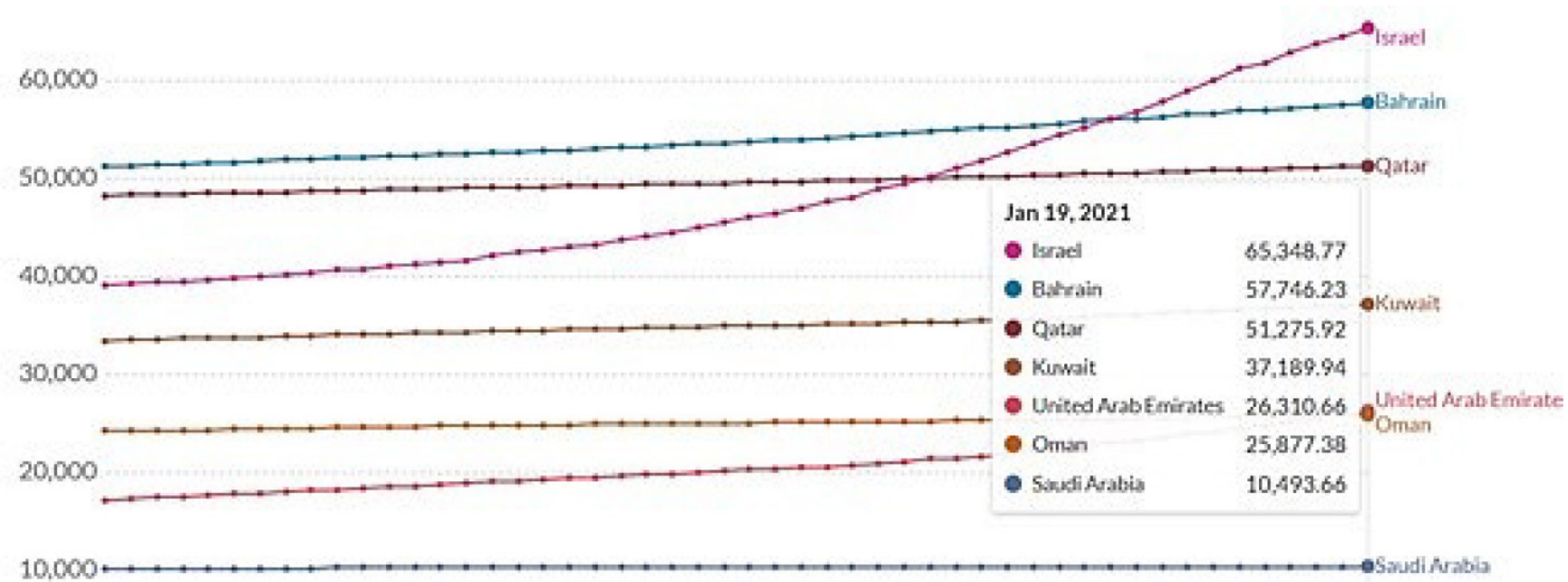

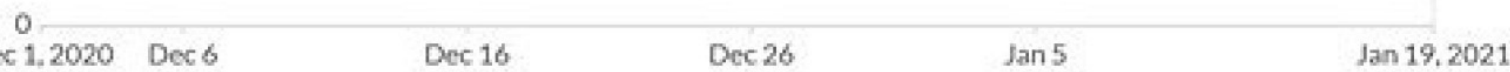

\section{$a$}

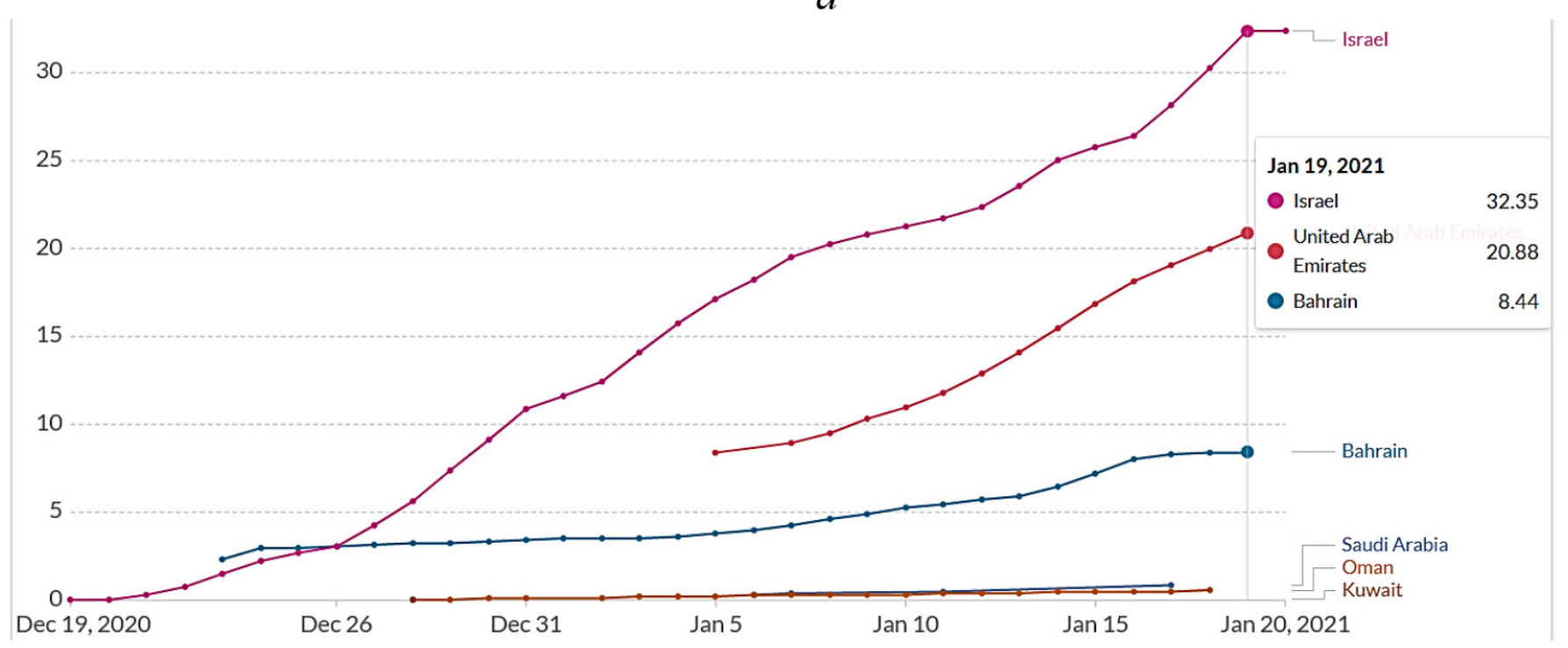

$b$

F I G U RE 3. Cumulative cases per million people (a) and vaccine doses administered per 100 people (b). Images from ourworldindata.org. CC BY.

2 doses, then $600 \mathrm{mg}$ PO BID + Camostat $200 \mathrm{mg}$ PO TID \pm nebulized Interferon Alpha or Interferon Beta (for 5 days), or Lopinavir-Ritonavir (200/50 mg) 2 tablets PO BID + Ribavirin $400 \mathrm{~m}$ PO BID for 7 days + nebulized Interferon, or Remdesivir $200 \mathrm{mg}$ IV on day 1 , followed by $100 \mathrm{mg}$ IV daily. Antibiotics may also be used if bacterial co-infection is suspected, plus anticoagulation and steroids when deemed necessary. Tocilizumab may be also considered in the case of the cytokine storm. Convalescent plasma is an additional experimental therapy [4].

\section{Discussion}

The latest share of the population infected (confirmed cases), an indicator of the extension of the infection, is $6.1 \%$ for
Belgium and Bahrein, 5.6\% for the UK, 5.3\% for Qatar, $4.2 \%$ for Italy, $3.1 \%$ for the UAE, and $0.1 \%$ for Australia. With about the same shares of the population infected, the case fatality rates are very different. The latest case fatality rate in the UAE is $0.3 \%$, and neighboring countries such as Qatar and Bahrain have $0.2 \%$ and $0.4 \%$ respectively. The case fatality rate of the UK is $2.8 \%$, Belgium $3 \%$, Australia $3.2 \%$, and Italy $3.5 \%$. While not only therapies contribute to this huge difference, however, therapies also contribute to making this difference.

The use of antivirals during the initial stages of the infection (asymptomatic at risk, and mild cases) is likely the main reason behind the reduced number of fatalities of countries such as the UAE, Bahrein, and Qatar. It is certainly during the first phase of Covid-19, that viral-induced effects are prominent, and the 
antiviral therapies may prevent the build-up of the viral load.

Nebulized interferon is optional in late cases, pneumonia for 7 days, or severe pneumonia/critically ill patients for 10 days [4]. For severe pneumonia/critically ill patients for 10 days, Tocilizumab may be considered in case of cytokine storm [4]. The antivirals are still administered in these late cases [4].

The UAE protocol is based on the available therapeutic options and specific experience. Other antivirals such as umifenovir, oseltamivir, or azithromycin, which are not mentioned in [4], may certainly be valuable.

Information about virus pathophysiology, if needed, can be found in $[5,6]$.

Antiviral drugs inhibit viral entry, viral membrane fusion, and endocytosis, or the activity of the 3-chymotrypsin-like protease and the RNA-dependent RNA polymerase [7, 8].

Favipiravir is an oral antiviral RNA polymerase inhibitor [912], of formula $\mathrm{C}_{5} \mathrm{H}_{4} \mathrm{FN}_{3} \mathrm{O}_{2}$.

Camostat mesylate is an oral antiviral serine protease inhibitor [13, 14].

Lopinavir-Ritonavir, of formula $\mathrm{C}_{37} \mathrm{H}_{48} \mathrm{~N}_{6} \mathrm{O}_{5} \mathrm{~S}_{2}$, is an antiretroviral medication for the treatment and prevention of HIV/AIDS repurposed for Covid-19 [15-17].

Ribavirin, of formula $\mathrm{C}_{8} \mathrm{H}_{12} \mathrm{~N}_{4} \mathrm{O}_{5}$, is an antiviral previously considered for RSV infection, hepatitis $\mathrm{C}$ and some viral hemorrhagic fevers, then repurposed for Covid-19 [18-20].

Remdesivir, of formula $\mathrm{C}_{27} \mathrm{H}_{35} \mathrm{~N}_{6} \mathrm{O}_{8} \mathrm{P}$, is a broad-spectrum antiviral repurposed for Covid-19 [21-23].

Immune-based-therapy is centered on human blood-derived products and immunomodulatory therapies [24-31].

Interferons are a family of cytokines with indirect antiviral properties [32-34]. They are signaling proteins made and released by host cells in response to viral infection. interferons are cytokines, molecules used for communication between cells to trigger protective defenses.

Corticosteroids, as well as interleukin inhibitors, interferons, kinase inhibitors, are more used as anti-inflammatory treatments, rather than antivirals.

Immune-based therapies (interferon) are used in [4] most as therapies supporting antiviral therapies, rather than standalone options, for anti-inflammatory effects.

Other adjunctive therapies are possible to prevent and/or treat the infection or its complications, and we mention antithrombotic therapy [35, 36], Vitamin C [37, 38], Vitamin D $[39,40]$, and Zinc [41, 42] as additional lines of actions.

One of the antiviral agents of [4] is CQ/HCQ, which has been at the center of great controversy. Statistically, from the analysis of the works published so far, 230 total, of which 165 peer-reviewed [43], almost $100 \%$ of the works support early treatment use. Controversial results are limited to the late cases, with however $74 \%$ of the studies still reporting positive effects. The use in early stages, generally supported in the literature [44-52], is also adopted with success in [4].

The protocols of neighboring countries (such as Bahrein and Qatar) also use CQ/HCQ with similarly positive results.

The work [53] that determined the ban of CQ/HCQ in the western countries, was a late study on extremely ill patients that were administered very high HCQ dosage, $1.6 \mathrm{~g}$ in the first 24 hours, $9.6 \mathrm{~g}$ total over 10 days, with no mention of weight or obesity status of patients to adjust for toxicity. Almost all excess mortality was from ventilated patients who received these extremely high doses for too many days. Asymptomatic Covid-19 cases or Covid-19 cases without pneumonia for 5 days, receive half these dosages over half the total time in [4]. Severe pneumonia/critically ill patients do not receive CQ/HCQ in [4].

While safety vs. efficacy of antivirals has to be considered carefully, without any doubt antivirals are determinant in the fight against Covid-19 infection.

Finally, the UAE is in second place in the world for administered vaccine doses after Israel. The curve of new cases is not decelerating, but accelerating phased on the administration of vaccines, Fig. 3.a,b. While this occurrence may be only temporary, and correlation is not causation, if antivirals are "imperfect", vaccines also have issues.

While vaccine doses are not the same for vaccinated people, as two shots are usually needed, a significant amount of the population of Israel and the UAE starts to be vaccinated, but the number of cases does not stop growing.

While the WHO [54] states that remdesivir, hydroxychloroquine, lopinavir/ritonavir, and interferon "had little or no effect on overall mortality, initiation of ventilation and duration of hospital stay in hospitalized patients" and "only corticosteroids have been proven effective against severe and critical Covid19" cases, many countries such as the UAE (and Qatar or Bahrein) are using these "imperfect" antivirals while measuring reduced case fatality rates vs. countries that rigidly follow the WHO guidelines.

As it is emerging from the UAE and Israel, where mass vaccinations are ongoing, vaccines are not perfect. In the news, thousands of Israelis tested positive for Covid-19 despite receiving the vaccine $[55,56]$. As of January 20, 2021, 189,000 people were tested for Covid-19 after being vaccinated. 12,400 or $6.6 \%$ returned a positive result. This is above the levels in the general population at about $5 \%$. The majority was infected shortly after the first of the two shots of the specific vaccine (by Pfizer/BioNTech). However, 1,410 returned a positive result 2 weeks after the first injection, and 69 returned a positive result after both shots of the vaccine. How many people were tested after two shots are unknown.

As of January 20, 2021, 2.15 million people have been vaccinated in Israel over roughly one month. 300,000 of them have received a second dose. The population is 9 million. These vaccinations haven't produced so far any benefit in the curve of newly infected cases.

Further comments are presently impossible, because of the limited data available subjected to continuous update.

The vaccine Israel uses is the Pfizer/BioNTech vaccine. A small number of doses from Moderna have also been administered. Both the Moderna and the Pfizer/BioNTech vaccines require two shots, priming, and booster shots. The interval between shots is 28 days for Moderna and 21 days for the Pfizer vaccine. The three vaccines in the UAE are the PfizerBioNTech, the Sinopharm, and the Sputnik V. Sinopharm and Sputnik V are also two doses.

Regarding the very latest vaccination number, the statistic of ourworldindata now includes the number of fully and partially vaccinated.

As of January 30, 2021, in Israel, the cumulative Covid-19 
vaccination doses administered per 100 people are 54.72. On December 20, 2020, they were 0.09 . The positive rate (the share of Covid-19 tests that are positive) is 5.7\% as of January 29, 2021. On December 20, 2020, it was 5.1\%. The tendency was decreasing till December 23, 2020, when the tendency changed. A flat $5 \%$ positive rate was measured till December 31,2020 . Then, since January 1, 2021, the positive rate started to grow. As of January 30, 2021, in Israel, 19.98\% of the population has received full vaccination, and $34.74 \%$ of the population received at least one vaccine dose.

As of January 31, 2021, in the UAE the cumulative Covid19 vaccination doses administered per 100 people are 33.71 . On January 5, 2021, they were 8.35. While no prior data is available, vaccinations started December 15, 2020. As of January 29,2021 , the positive rate is $1.2 \%$. On December 20 , 2020 , it was $1.0 \%$. As of January 31, 2021, in the UAE, $2.53 \%$ of the population has received full vaccination, and $31.18 \%$ of the population received at least one vaccine dose. Same as Israel, the tendency in the positive rate was a decreasing positive rate till December 27, 2020, when a slightly less than unity positive rate was measured till December 31, 2020. Then, since January 1, 2021, the positive rate started to grow.

The number of tests is much larger in the UAE, while the severity of the outbreak is much worse in Israel.

On January 15, 2021, the UAE was conducting 14.76 tests per 1,000 people, and Israel 12.76. Then, Israel decided to reduce the number of tests, while the UAE decided the opposite. On January 29, 2021, the UAE has performed 17.23 tests per 1,000 people, while Israel has reduced the number of tests to 7.75. The reduction in the number of tests started when the press raised concern about the growing number of cases.

Thus, while vaccinations may certainly help to resolve the Covid-19 emergency, unlikely they will completely address the issue. It is therefore of paramount importance to improve the therapies for those infected, learning from the best performing protocols under objective parameters.

\section{Conclusions}

The therapeutic approach followed in the UAE seems particularly successful, as the cumulative fatality rate, that however also depends on many other factors, is about 10 times smaller than the one in Italy, Belgium, Australia, or the United Kingdom. The use of antivirals in every phase of Covid-19 infection, but especially asymptomatic and mild to medium cases, is likely one driver of reduced fatalities. Different opportunities are available in the UAE, also based on combining different therapeutic agents. The best treatment of ARDS, often leading to fatalities, is to prevent this occurrence in the early stages. This depends on the way asymptomatic to mild cases are treated, before becoming critical. The use of antiviral agents is determinant to reduce the fatalities of Covid-19 infection. This is the lesson we learn from comparing cases, fatalities, and therapies of different countries.

\section{AUTHOR CONTRIBUTIONS}

Single author paper.

\section{ACKNOWLEDGMENT}

Thanks to all the peer reviewers for their opinions and suggestions.

\section{FUNDING}

This research received no specific grant from any funding agency in the public, commercial, or not-for-profit sectors.

\section{CONFLICT OF INTEREST}

The authors declare that there is no conflict of interest regarding the publication of this article.

\section{DATA AVAILABILITY}

The data used to support the findings of this study are available from the listed references.

\section{REFERENCES}

[1] Wang Y, Zhang L, Sang L, Ye F, Ruan S, Zhong B, et al. Kinetics of viral load and antibody response in relation to COVID-19 severity. Journal of Clinical Investigation. 2020; 130: 5235-5244.

[2] Xu T, Chen C, Zhu Z, Cui M, Chen C, Dai H, et al. Clinical features and dynamics of viral load in imported and non-imported patients with COVID-19. International Journal of Infectious Diseases. 2020; 94: 6871.

[3] Westblade LF, Brar G, Pinheiro LC, Paidoussis D, Rajan M, Martin P, et al. SARS-CoV-2 viral load predicts mortality in patients with and without cancer who are hospitalized with COVID-19. Cancer Cell. 2020; 38: 661671.e2.

[4] doh.gov.ae/-/media/7BD7B077D8F846B48A70C5872902DD1C.ashx

[5] Kumar R, Sharma A, Srivastava JK, Siddiqui MH, Uddin MS, Aleya L. Hydroxychloroquine in COVID-19: therapeutic promises, current status, and environmental implications. Environmental Science and Pollution Research. 2021; 1-14.

[6] Kumar R, Srivastava JK, Singh R, Siddiqui MH, Mansouri RA, Abdulhakim JA, et al. Available Compounds with Therapeutic Potential against COVID-19: antimicrobial therapies, supportive care and probable vaccines. Frontiers in pharmacology. 2020; 11: 1592.

[7] Sanders JM, Monogue ML, Jodlowski TZ, Cutrell JB. Pharmacologic treatments for coronavirus disease 2019 (COVID-19): a review. Journal of the American Medical Association. 2020; 323: 1824-1836.

[8] Siddiqi HK, Mehra MR. COVID-19 illness in native and immunosuppressed states: a clinical-therapeutic staging proposal. The Journal of Heart and Lung Transplantation. 2020; 39: 405-407.

[9] Boretti A. Favipiravir use for SARS CoV-2 infection. Pharmacological Reports. 2020; 72: 1542-1552.

[10] Cai Q, Yang M, Liu D, Chen J, Shu D, Xia J, et al. Experimental Treatment with Favipiravir for COVID-19: an open-label control study. Engineering. 2020; 6: 1192-1198.

[11] Lou Y, Liu L, Yao H, Hu X, Su J, Xu K, et al. Clinical Outcomes and plasma concentrations of baloxavir marboxil and favipiravir in COVID19 patients: an exploratory randomized, controlled trial. European Journal of Pharmaceutical Sciences. 2021; 157: 105631.

[12] Yamamura H, Matsuura H, Nakagawa J, Fukuoka H, Domi H, Chujoh S. Effect of favipiravir and an anti-inflammatory strategy for COVID-19. Critical Care. 2020; 24: 413.

[13] Breining P, Frølund AL, Højen JF, Gunst JD, Staerke NB, Saedder E, et al. Camostat mesylate against SARS-CoV-2 and COVID-19-rationale, dosing and safety. Basic \& Clinical Pharmacology \& Toxicology. 2021; 128: 204-212.

[14] Hofmann-Winkler H, Moerer O, Alt-Epping S, Bräuer A, Büttner B, 
Müller M, et al. Camostat mesylate may reduce severity of coronavirus disease 2019 sepsis: a first observation. Critical Care Explorations. 2020; 2: $\mathrm{e} 0284$.

[15] Cao B, Wang Y, Wen D, Liu W, Wang J, Fan G, et al. A trial of lopinavirritonavir in adults hospitalized with severe COVID-19. New England Journal of Medicine. 2020; 382: 1787-1799.

[16] Lim J, Jeon S, Shin HY, Kim MJ, Seong YM, Lee WJ, et al. Case of the index patient who caused tertiary transmission of COVID-19 infection in Korea: the application of lopinavir/ritonavir for the treatment of COVID19 infected pneumonia monitored by quantitative RT-PCR. Journal of Korean Medical Science. 2020; 35: e79.

[17] Horby PW, Mafham M, Bell JL, Linsell L, Staplin N, Emberson J, et al. Lopinavir-ritonavir in patients admitted to hospital with COVID-19 (RECOVERY): a randomised, controlled, open-label, platform trial. The Lancet. 2020; 396: 1345-1352.

[18] Khalili JS, Zhu H, Mak NSA, Yan Y, Zhu Y. Novel coronavirus treatment with ribavirin: groundwork for an evaluation concerning COVID-19. Journal of Medical Virology. 2020; 92: 740-746.

[19] Tong S, Su Y, Yu Y, Wu C, Chen J, Wang S, et al. Ribavirin therapy for severe COVID-19: a retrospective cohort study. International Journal of Antimicrobial Agents. 2020; 56: 106114.

[20] Eslami G, Mousaviasl S, Radmanesh E, Jelvay S, Bitaraf S, Simmons B, et al. The impact of sofosbuvir/daclatasvir or ribavirin in patients with severe COVID-19. Journal of Antimicrobial Chemotherapy. 2020; 75: 3366-3372.

[21] Beigel JH, Tomashek KM, Dodd LE, Mehta AK, Zingman BS, Kalil AC, et al. Remdesivir for the treatment of Covid-19-final report. New England Journal of Medicine. 2020; 383: 1813-1826.

[22] Al-Tawfiq JA, Al-Homoud AH, Memish ZA. Remdesivir as a possible therapeutic option for the COVID-19. Travel Medicine and Infectious Disease. 2020; 34: 101615.

[23] McCreary EK, Angus DC. Efficacy of remdesivir in COVID-19. Journal of the American Medical Association. 2020; 324: 1041-1042.

[24] Zhong J, Tang J, Ye C, Dong L. The immunology of COVID-19: is immune modulation an option for treatment? The Lancet Rheumatology. 2020; 2: e428-e436.

[25] Ellison-Hughes GM, Colley L, O’Brien KA, Roberts KA, Agbaedeng TA, Ross MD. The role of MSC therapy in attenuating the damaging effects of the cytokine storm induced by COVID-19 on the heart and cardiovascular system. Frontiers in cardiovascular medicine. 2020; 7: 602183.

[26] Marovich M, Mascola JR, Cohen MS. Monoclonal antibodies for prevention and treatment of COVID-19. Journal of the American Medical Association. 2020; 324: 131-132.

[27] Monteagudo LA, Boothby A, Gertner E. Continuous intravenous anakinra infusion to calm the cytokine storm in macrophage activation syndrome. ACR Open Rheumatology. 2020; 2: 276-282.

[28] Monteagudo LA, Boothby A, Gertner E. Continuous intravenous anakinra infusion to calm the cytokine storm in macrophage activation syndrome. ACR Open Rheumatology. 2020; 2: 276-282.

[29] Xu X, Han M, Li T, Sun W, Wang D, Fu B, et al. Effective treatment of severe COVID-19 patients with tocilizumab. Proceedings of the National Academy of Sciences. 2020; 117: 10970-10975.

[30] Zhou Q, Wei XS, Xiang X, Wang X, Wang ZH, Chen V, et al. Interferon$\alpha 2 \mathrm{~b}$ treatment for COVID-19. 2020; MedRxiv.

[31] Cao Y, Wei J, Zou L, Jiang T, Wang G, Chen L, et al. Ruxolitinib in treatment of severe coronavirus disease 2019 (COVID-19): a multicenter, single-blind, randomized controlled trial. Journal of Allergy and Clinical Immunology. 2020; 146: 137-146.e3.

[32] Lee JS, Shin E. The type I interferon response in COVID-19: implications for treatment. Nature Reviews Immunology. 2020; 20: 585-586.

[33] Zhou Q, Chen V, Shannon CP, Wei XS, Xiang X, Wang X, et al. Interferon- $\alpha 2 \mathrm{~b}$ treatment for COVID-19. Frontiers in Immunology. 2020; 11: 1061 .

[34] Davoudi-Monfared E, Rahmani H, Khalili H, Hajiabdolbaghi M, Salehi $\mathrm{M}$, Abbasian L, et al. A randomized clinical trial of the efficacy and safety of interferon $\beta$-1a in treatment of severe COVID-19. Antimicrobial Agents and Chemotherapy. 2020; 64: e01061-20.

[35] Bikdeli B, Madhavan MV, Jimenez D, Chuich T, Dreyfus I, Driggin E, et al. COVID-19 and thrombotic or thromboembolic disease: implications for prevention, antithrombotic therapy, and follow-up. Journal of the
American College of Cardiology. 2020; 75: 2950-2973.

[36] Chaudhary R, Kreutz RP, Bliden KP, Tantry US, Gurbel PA. Personalizing antithrombotic therapy in COVID-19: role of thromboelastography and thromboelastometry. Thrombosis and Haemostasis. 2020; 120: 15941596.

[37] Boretti A, Banik BK. Intravenous vitamin C for reduction of cytokines storm in acute respiratory distress syndrome. PharmaNutrition. 2020; 12 : 100190.

[38] Cheng RZ. Can early and high intravenous dose of vitamin $\mathrm{C}$ prevent and treat coronavirus disease 2019 (COVID-19)? Medicine in Drug Discovery. 2020; 5: 100028 .

[39] Cereda E, Bogliolo L, de Stefano L, Caccialanza R. A brief discussion of the benefit and mechanism of vitamin $\mathrm{D}$ supplementation on coronavirus disease 2019. Current Opinion in Clinical Nutrition \& Metabolic Care. 2021; 24: 102-107.

[40] Meltzer DO, Best TJ, Zhang H, Vokes T, Arora V, Solway J. Association of vitamin D status and other clinical characteristics with COVID-19 test results. JAMA Network Open. 2020; 3: e2019722.

[41] Anuk AT, Polat N, Akdas S, Erol SA, Tanacan A, Biriken D, et al. The relation between trace element status (zinc, copper, magnesium) and clinical outcomes in COVID-19 infection during pregnancy. Biological trace element research. 2020; 1-10.

[42] Wessels I, Rolles B, Rink L. The potential impact of zinc supplementation on COVID-19 pathogenesis. Frontiers in Immunology. 2020; 11: 1712.

[43] HCQ for COVID-19. Available at: c19study.com/.

[44] Procter BC, Ross C, Pickard V, Smith E, Hanson C, McCullough PA. Clinical outcomes after early ambulatory multidrug therapy for highrisk SARS-CoV-2 (COVID-19) infection. Reviews in Cardiovascular Medicine. 2020; 21: 611-614.

[45] Su Y, Ling Y, Ma Y, Tao L, Miao Q, Shi Q, et al. Efficacy of early hydroxychloroquine treatment in preventing COVID-19 pneumonia aggravation, the experience from Shanghai, China. BioScience Trends. 2020; 14: 408-414.

[46] Sogut O, Can MM, Guven R, Kaplan O, Ergenc H, Umit TB, et al. Safety and efficacy of hydroxychloroquine in 152 outpatients with confirmed COVID-19: a pilot observational study. The American Journal of Emergency Medicine. 2021; 40: 41-46.

[47] Agusti A, Guillen E, Ayora A, Anton A, Aguilera C, Vidal X, et al. Efficacy and safety of hydroxychloroquine in healthcare professionals with mild SARS-CoV-2 infection: prospective, non-randomized trial. Enfermedades Infecciosas Y Microbiología ClíNica. 2020; S0213005X(20)30413-4.

[48] Omrani AS, Pathan SA, Thomas SA, Harris TRE, Coyle PV, Thomas $\mathrm{CE}$, et al. Randomized double-blinded placebo-controlled trial of hydroxychloroquine with or without azithromycin for virologic cure of non-severe COVID-19. EClinicalMedicine. 2020; 29-30: 100645.

[49] Szente Fonseca SN, de Queiroz Sousa A, Wolkoff AG, Moreira MS, Pinto BC, Valente Takeda CF, et al. Risk of hospitalization for COVID19 outpatients treated with various drug regimens in Brazil: comparative analysis. Travel Medicine and Infectious Disease. 2020; 38: 101906.

[50] Derwand R, Scholz M, Zelenko V. COVID-19 outpatients: early riskstratified treatment with zinc plus low-dose hydroxychloroquine and azithromycin: a retrospective case series study. International Journal of Antimicrobial Agents. 2020; 56: 106214.

[51] Prodromos C, Rumschlag T. Hydroxychloroquine is effective, and consistently so used early, for COVID-19: a systematic review. New microbes and new infections. 2020; 38: 100776.

[52] Hong KS, Jang JG, Hur J, Lee JH, Kim HN, Lee W, et al. Early hydroxychloroquine administration for rapid severe acute respiratory syndrome coronavirus 2 eradication. Infection \& Chemotherapy. 2020; 52: 396.

[53] RECOVERY. Randomised evaluation of COVID-19 therapy. 2020. Available at: www.recoverytrial.net/files/protocolarchive/recovery-protocol-v4-0-2020-04-14.pdf (Accessed: 30 January 2021).

[54] WHO Solidarity Trial Consortium, 2020. Repurposed antiviral drugs for COVID-19-interim WHO SOLIDARITY trial results. New England Journal of Medicine. 2021; 384:497-511.

[55] RT question more. Thousands of Israelis test POSITIVE for Covid-19 despite receiving Pfizer/BioNTech jab. 2021. Available at: www.rt. 
com/news/513139-israel-pfizer-vaccine-coronavirus/ (Accessed: 30 January 2021).

[56] Efrati I, Linder R. Thousands of Israelis tested positive for coronavirus after first vaccine shot. Available at: www.haaretz.com/israelnews/thousands-of-israelis-tested-positive-forcoronavirus-after-first-vaccine-shot-1.9462478.
How to cite this article: Alberto Boretti. Analysis of the performances of the Covid-19 therapeutic approaches in the United Arab Emirates. Signa Vitae. 2021;17(3):256-263. doi: $10.22514 /$ sv.2021.041. 